Global Journal of Pure and Applied Mathematics.

ISSN 0973-1768 Volume 11, Number 4 (2015), pp. 2347-2356

(C) Research India Publications

http://www.ripublication.com/gjpam.htm

\title{
Visualization of classified data with kernel principal component analysis
}

\author{
Sonna Ariyanto Lobo, Siswadi and Toni Bakhtiar \\ Department of Mathematics, \\ Bogor Agricultural University, \\ Jl. Meranti, Kampus IPB Dramaga, \\ Bogor 16880, Indonesia. \\ E-mail: zonnalobo@gmail.com, siswadi@inrr.org,tbakhtiar@ipb.ac.id
}

\begin{abstract}
Kernel Principal Component Analysis (Kernel PCA) is a generalization of the ordinary PCA which allows mapping the original data into a high-dimensional feature space. The mapping is expected to address the issues of nonlinearity among variables and separation among classes in the original data space. The key problem in the use of kernel PCA is the parameter estimation used in kernel functions that so far has not had quite obvious guidance, where the parameter selection mainly depends on the objectivity of the research. This study exploited the use of Gaussian kernel function and focused on the ability of kernel PCA in visualizing the separation of the classified data. Assessments were undertaken based on misclassification obtained by Fisher Discriminant Linear Analysis of the first two principal components. This study results suggest for the visualization of kernel PCA by selecting the parameter in the interval between the closest and the furthest distances among the objects of original data is better than that of ordinary PCA.
\end{abstract}

AMS Subject Classification: 62H25, 62H30, 91C20.

Keywords: Kernel PCA, Gaussian kernel function, data visualization, Fisher discriminant linear analysis.

\section{Introduction}

\subsection{Background}

Visualization is engineered in making drawings, diagrams or animations to the appearance of an information. In statistics, visualization is often used in exploratory data 
analysis as an approach in analyzing data to summarize the main characteristics of the data [4]. The primary objective of data visualization is to communicate information clearly and effectively to users through graphs, plots and tables. It makes the complex data to be more accessible, understood and used. For data with dimension greater than three, the visualization cannot be done directly by using the data objects in the ordinary space, otherwise, it can be done by using Principal Component Analysis (PCA).

PCA is probably the oldest and best known of the techniques of multivariate analysis. The central idea of PCA is to reduce the dimensionality of a data set in which it has correlation among variables, while retaining as much as possible of the variation present in the data set [8]. The new variables that represent the data with smaller dimension but uncorrelated is called principal components. From the algebraic point of view, a principal component is obtained by a linear combination of the original variables which have the largest variance in sequence and do not have correlation with the previous principal components. However, since PCA uses a linear combination of the variables to interpret the data, PCA could not model complex data with nonlinear relationships among variables. Therefore, Kernel Principal Component Analysis (Kernel PCA) was developed as the generalization of the ordinary PCA.

Implicitly, the kernel PCA is a nonlinear form of the ordinary PCA which maps the original data with nonlinear relationships among variables into a high-dimensional feature space. At the space, the data obtained has linear relationships among variables so that ordinary PCA can be applied [12]. For classified data, in addition to linear relationships among the variables, the separation among the classes become a problem in itself. The data in the feature space is expected to be separately marked by having few misclassification. By using kernel function which is a standard dot product in the feature space, the data is mapped from original space into the feature space. The success of kernel PCA is determined by kernel function used. One of the kernel functions that is widely popular is Gaussian kernel function which having form

$$
\kappa\left(\mathbf{x}_{i}, \mathbf{x}_{j}\right)=e^{-\frac{\left\|\mathbf{x}_{i}-\mathbf{x}_{j}\right\|^{2}}{2 \sigma^{2}}},
$$

where $\mathbf{x}$ is a vector of data objects and $\sigma$ is a parameter [13]. The ability of Gaussian kernel function to map the classified data into feature space to obtain the separation among classes is very dependent on the parameter. Many studies have been done by using Gaussian kernel function to reduce the dimensionality of the data $[1,9,10,11,14,16]$. Each study has its own method in selecting the parameter, depends on the research objective. Until now, method to choose the optimal parameters is still an ongoing research topic with no conclusive solution.

This study aims to assess the selection method for parameter $\sigma$ of Gaussian kernel function in visualizing the classified data with minimum misclassification. In addition, the visualization results by kernel PCA is also compared with those by ordinary PCA based on the respecting misclassification. We exploited Fisher linear discriminant analysis to show the linearly separation of the data in the feature space. We present a short review of kernel PCA and Fisher discriminant linear analysis in the next subsections. 


\subsection{Kernel PCA}

Consider a data set $\mathbf{X}_{n \times p}=\left(\mathbf{x}_{1}, \mathbf{x}_{2}, \ldots, \mathbf{x}_{n}\right)^{T}$, where $\mathbf{x}_{i} \in \mathbb{R}^{p}$ and assume that we have a nonlinear transformation $\phi\left(\mathbf{x}_{i}\right)$, which transforms the original data into $p_{f}$-dimensional feature space, where usually $p_{f} \gg p$. We define the data centered in the feature space by $\tilde{\phi}\left(\mathbf{x}_{i}\right)$, from which the covariance matrix $\mathbf{C}$ in $\mathbb{R}^{p_{f}}$ is given by

$$
\mathbf{C}=\frac{1}{n-1} \sum_{i=1}^{n} \tilde{\phi}\left(\mathbf{x}_{i}\right) \tilde{\phi}\left(\mathbf{x}_{i}\right)^{T} .
$$

Similar to the ordinary PCA [8], we need to find eigenvectors $\mathbf{v}$ and eigenvalues $\lambda$ by solving eigen problem $\mathbf{C v}=\lambda \mathbf{v}$. But, it can be extremely costly and inefficient because $p_{f}$ could be so big. Fortunately, we can use kernel method to simplify [12]. Since the dot product in the feature space is defined by $\left\langle\tilde{\phi}\left(\mathbf{x}_{i}\right), \tilde{\phi}\left(\mathbf{x}_{i}\right)\right\rangle=\tilde{\phi}\left(\mathbf{x}_{i}\right)^{T} \tilde{\phi}\left(\mathbf{x}_{i}\right)$, the eigen problem $\mathbf{C v}=\lambda \mathbf{v}$, using (1.2), can then be expressed by

$$
\frac{1}{n-1} \sum_{i=1}^{n} \tilde{\phi}\left(\mathbf{x}_{i}\right) \tilde{\phi}\left(\mathbf{x}_{i}\right)^{T} \mathbf{v}^{m}=\lambda_{m} \mathbf{v}^{m}
$$

which implies that $\mathbf{v}^{m}$ corresponding to $\lambda_{m}, m=1,2, \ldots, r$ with $r=\operatorname{rank}(\mathbf{X})$ and $\lambda_{1} \geq \lambda_{2} \geq \cdots \lambda_{r}>0$ is a linear combination of $\left\{\tilde{\phi}\left(\mathbf{x}_{1}\right), \tilde{\phi}\left(\mathbf{x}_{2}\right), \ldots, \tilde{\phi}\left(\mathbf{x}_{n}\right)\right\}$. Thus, we can expand $\mathbf{v}^{m}$ as follows

$$
\mathbf{v}^{m}=\sum_{i=1}^{n} \alpha_{i}^{m} \tilde{\phi}\left(\mathbf{x}_{i}\right), \quad \alpha_{i}^{m}=\frac{\left\langle\tilde{\phi}\left(\mathbf{x}_{i}\right), \mathbf{v}^{m}\right\rangle}{(n-1) \lambda_{m}} .
$$

Now by using (1.3) and (1.4), the eigen problem is equivalent to

$$
\left\langle\tilde{\phi}\left(\mathbf{x}_{j}\right), \mathbf{C v}^{m}\right\rangle=\left\langle\tilde{\phi}\left(\mathbf{x}_{j}\right), \lambda_{m} \mathbf{v}^{m}\right\rangle,
$$

for $j=1,2, \ldots, n$, which means

$$
\frac{1}{n-1} \sum_{i=1}^{n} \sum_{k=1}^{n} \alpha_{k}^{m}\left\langle\tilde{\phi}\left(\mathbf{x}_{i}\right), \tilde{\phi}\left(\mathbf{x}_{k}\right)\right\rangle\left\langle\tilde{\phi}\left(\mathbf{x}_{j}\right), \tilde{\phi}\left(\mathbf{x}_{i}\right)\right\rangle=\lambda_{m} \sum_{k=1}^{n} \alpha_{k}^{m}\left\langle\tilde{\phi}\left(\mathbf{x}_{j}\right), \tilde{\phi}\left(\mathbf{x}_{k}\right)\right\rangle .
$$

According to [3], by defining kernel matrix $\tilde{\mathbf{K}}_{n \times n}$ as follows

$$
\tilde{\mathbf{K}}_{i j}=\kappa\left(\mathbf{x}_{i}, \mathbf{x}_{j}\right)-\frac{1}{n} \sum_{j=1}^{n} \kappa\left(\mathbf{x}_{i}, \mathbf{x}_{j}\right)-\frac{1}{n} \sum_{i=1}^{n} \kappa\left(\mathbf{x}_{i}, \mathbf{x}_{j}\right)+\frac{1}{n^{2}} \sum_{i=1}^{n} \sum_{j=1}^{n} \kappa\left(\mathbf{x}_{i}, \mathbf{x}_{j}\right),
$$

we can write (1.5) as

$$
\tilde{\mathbf{K}}^{2} \alpha^{m}=\lambda_{m} \tilde{\mathbf{K}} \alpha^{m} \Leftrightarrow \tilde{\mathbf{K}} \alpha^{m}=\lambda_{m} \alpha^{m} .
$$


Furthermore, we normalize the eigenvector $\mathbf{v}^{m}$ in order to have $\tilde{\mathbf{v}}^{m}$ where $\left\langle\tilde{\mathbf{v}}^{m}, \tilde{\mathbf{v}}^{m}\right\rangle=1$, then we obtain

$$
\tilde{\mathbf{v}}^{m}=\frac{1}{\sqrt{(n-1) \lambda_{m}}} \sum_{i=1}^{n} \alpha_{i}^{m} \tilde{\phi}\left(\mathbf{x}_{i}\right)=\sum_{i=1}^{n} \tilde{\alpha}_{i}^{m} \tilde{\phi}\left(\mathbf{x}_{i}\right) .
$$

For principal components extraction in the feature space, we compute the projections of $\tilde{\phi}(\mathbf{x})$ toward eigenvectors $\mathbf{v}^{m}$ in feature space according to

$$
\left\langle\mathbf{v}^{m}, \tilde{\phi}(\mathbf{x})\right\rangle=\sum_{i=1}^{n} \alpha_{i}^{m} \kappa\left(\mathbf{x}_{i}, \mathbf{x}\right),
$$

or simply in a matrix notation:

$$
\tilde{\Phi} \tilde{\mathbf{V}}=\tilde{\mathbf{K}} \tilde{\mathbf{A}}
$$

where we denote $\tilde{\Phi}_{n \times n_{f}}=\left(\tilde{\phi}\left(\mathbf{x}_{1}\right), \tilde{\phi}\left(\mathbf{x}_{2}\right), \ldots, \tilde{\phi}\left(\mathbf{x}_{n}\right)\right)^{T}, \tilde{\mathbf{V}}_{p_{f} \times r}=\left(\tilde{\mathbf{v}}^{1}, \tilde{\mathbf{v}}^{2}, \ldots, \tilde{\mathbf{v}}^{r}\right)$ and $\tilde{\mathbf{A}}_{n \times r}=\left(\tilde{\alpha}^{1}, \tilde{\alpha}^{2}, \ldots, \tilde{\alpha}^{r}\right)[10]$.

\subsection{Fisher Discriminant Linear Analysis}

Discriminant analysis is a multivariate technique concerned with separating distinct sets of objects and with allocating new objects to previously defined classes [7]. In the other words, discriminant analysis is used to classify an input vector $\mathbf{x}$ and allocating it into one of the $K$ data classes $\pi_{k}(k=1,2, \ldots, K)$. Suppose given $n$ training data objects collected in $\mathbf{X}_{n \times p}$ which consists of $K$ data classes. Each class of the data is assumed to be normally distributed with the mean vector and covariance matrix for class $\pi_{k}$ are $\boldsymbol{\mu}_{k}$ and $\boldsymbol{\Sigma}_{k}$, respectively, estimated using maximum likelihood estimates by $\overline{\mathbf{x}}_{k}$ and $\mathbf{S}_{k}$. When the population covariance matrices in each class are equal, i.e., $\boldsymbol{\Sigma}_{j}=\boldsymbol{\Sigma}_{k}=\boldsymbol{\Sigma}$ for all $j \neq k$, then we can write the linear discriminant function $d_{k}$ as follows:

$$
d_{k}(\mathbf{x})=\boldsymbol{\mu}_{k}^{T} \boldsymbol{\Sigma}^{-1} \mathbf{x}-\frac{1}{2} \boldsymbol{\mu}_{k}^{T} \boldsymbol{\Sigma}^{-1} \boldsymbol{\mu}_{k}
$$

Equation (1.11) is known as Fisher Linear Discriminant Function, where the allocation of any vector object $\mathbf{x}$ into class $\pi_{k^{*}}$ is decided according to

$$
k^{*}=\underset{k=1, \ldots, K}{\operatorname{argmax}} d_{k}(\mathbf{x})
$$

The function of hyperplanes that separates $\pi_{k}$ and $\pi_{j}$ for all $k \neq j$ is given by

$$
\left(\boldsymbol{\mu}_{k}-\boldsymbol{\mu}_{j}\right)^{T} \boldsymbol{\Sigma}^{-1}\left(\mathbf{x}-\frac{1}{2}\left(\boldsymbol{\mu}_{k}+\boldsymbol{\mu}_{j}\right)\right)=0 .
$$

The classified data that can exactly be separated by linear decision surfaces is said to be linearly separable [3]. 
Table 1: Data description

\begin{tabular}{lccc}
\hline & \multicolumn{3}{c}{ Number of } \\
\cline { 2 - 4 } Data Set & Objects $(n)$ & Variables $(p)$ & Classes $(K)$ \\
\hline Wang synthesis & 1000 & 3 & 2 \\
SCCTS & 600 & 60 & 6 \\
Iris & 150 & 4 & 3 \\
Wine & 178 & 13 & 3 \\
\hline
\end{tabular}

Table 2: Misclassification of Fisher linear discriminant analysis

\begin{tabular}{cccccc}
\hline & \multicolumn{6}{c}{ Predicted Membership } & \\
\cline { 2 - 5 } Actual Membership & $\pi_{1}$ & $\pi_{2}$ & $\cdots$ & $\pi_{K}$ & Total \\
\hline$\pi_{1}$ & $n_{11}$ & $n_{12}$ & $\cdots$ & $n_{1 K}$ & $n_{1 \cdot}$ \\
$\pi_{2}$ & $n_{21}$ & $n_{22}$ & $\cdots$ & $n_{2 K}$ & $n_{2 .}$ \\
$\vdots$ & $\vdots$ & $\vdots$ & $\ddots$ & $\vdots$ & $\vdots$ \\
$\pi_{K}$ & $n_{K 1}$ & $n_{K 2}$ & $\cdots$ & $n_{K K}$ & $n_{K}$. \\
\hline Total & $n_{\cdot 1}$ & $n_{\cdot 2}$ & $\cdots$ & $n_{\cdot K}$ & $n . .=n$ \\
\hline
\end{tabular}

\section{Material and Method}

In this section we provided data sets used in the research and the proposed method for selecting the parameter of Gaussian kernel function.

\subsection{Data}

The data used in this study consists of two synthesis data sets, namely Wang synthesis data set [15] and Synthetic Control Chart Time Series (SCCTS) data set [2], and two real world data sets, namely Iris data set [5] and Wine data set [6]. Each data, except Iris data set, was standardized to eliminate the variance dominance of certain variables. The description of data's classes is shown in Table 1.

\subsection{Method}

In this research, we used the Gaussian kernel function to map the data from the original space to the feature space. As we know, Gaussian kernel function has a parameter $\delta$. The purpose in selecting this parameter is mainly motivated by misclassification minimization problem under Fisher linear discriminant analysis, where the misclassification chart is shown in Table 2. Total proportion of misclassification is then given by apparent error rate $\varepsilon$ as follows

$$
\varepsilon=\frac{n-\sum_{k=1}^{K} n_{k k}}{n}
$$

In kernel PCA, Gaussian kernel function (1.1) is used to compute the Gram matrix which is a dual form of the covariance matrix in the feature space [10]. Thus, $\sigma$ will 
determine the correlation of the data in the feature space. If $\sigma$ is too large compared to the distances between data objects, it will result matrix of ones as the Gram matrix so that the data objects in the feature space are concentrated at one point. As a consequence, the greater $\sigma$, the lesser variance of the data in the feature space. Conversely, if $\sigma$ is too small, then the Gram matrix becomes the identity matrix so that the data has no correlation in the feature space and the ordinary PCA becomes infeasible. Consequently, the smaller $\sigma$, the lesser correlation variables in the feature space. Therefore, we propose to select $\sigma$ in the interval of $\min \left\|\mathbf{x}_{i}-\mathbf{x}_{j}\right\| \leq \sigma \leq \max \left\|\mathbf{x}_{i}-\mathbf{x}_{j}\right\|$. The interval is then partitioned and every point is tested to get a point which provides $\sigma$ with the smallest $\varepsilon$. If there are several points exist, then $\sigma$ is selected according to the largest percentage of variance of the first two principal components. The number of partitions of the interval selected is based on the width of the interval. In this study, the interval was divided into 200 partitions. The algorithm for selecting $\sigma$ can be presented below.

Input: Matrix of classified data $\mathbf{X}_{n \times p}=\left(\mathbf{x}_{1}, \mathbf{x}_{2}, \ldots, \mathbf{x}_{n}\right)^{T}$.

1. Compute $\left\|\mathbf{x}_{i}-\mathbf{x}_{j}\right\|$ for all $i, j=1, \ldots, n$ and $i \neq j$.

2. Define interval $R:=\left[\min \left\|\mathbf{x}_{i}-\mathbf{x}_{j}\right\|, \max \left\|\mathbf{x}_{i}-\mathbf{x}_{j}\right\|\right]$.

3. Make $n_{p}$ partitions over $R$ to produce $\left\{\sigma_{1}, \sigma_{2}, \ldots, \sigma_{n_{p}+1}\right\}$.

4. For all $\sigma_{i}$ : (i) reduce $\mathbf{X}$ using (1.10) into the first two principal components, (ii) classify the principal components using (1.11), (iii) compute the apparent error rate using (2.1), (iv) $\sigma^{*}=\underset{i}{\operatorname{argmin}} \varepsilon\left(\sigma_{i}\right)$.

The parameter $\sigma^{*}$ obtained from the algorithm is then used for visualizing data using kernel PCA. In addition, the data is also visualized using ordinary PCA to be compared with kernel PCA based on the apparent error rate. The original data is also classified to inspect the linearly separable property of the original data in the original space.

\section{Experiments}

\subsection{Visualization of Wang Synthesis Data Set}

We used Wang synthesis data set to illustrate the effectiveness of the proposed method in selecting $\sigma$ used in kernel PCA to visualize data set. The data is assumed to be distributed on two concentric sphere surfaces. The total number of all data points is 1000, which are divided into two classes. The first class has 500 points on a sphere of radius 100 and the second class has 500 points on a sphere of radius 40 . In the spherical coordinate system, the inclination $\theta$ is uniformly distributed in $[0, \pi]$ and the azimuth $\psi$ is uniformly distributed in $[0,2 \pi)$ for both classes. Further, the all data points are transformed to cartesian coordinate system. So, we have the observations of the data points as $(x, y, z)$ and then by a Gaussian noise of standard deviation 1, all observations are perturbed. Visualization of the data consists of two balls shown in Figure 1, which 

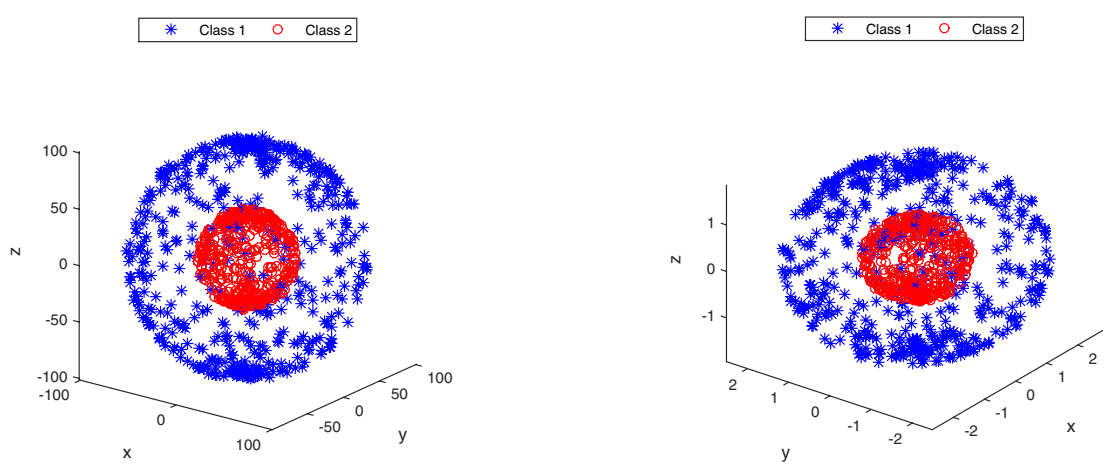

Figure 1: Visualization of Wang synthetic data set: original (left) and standardized (right).
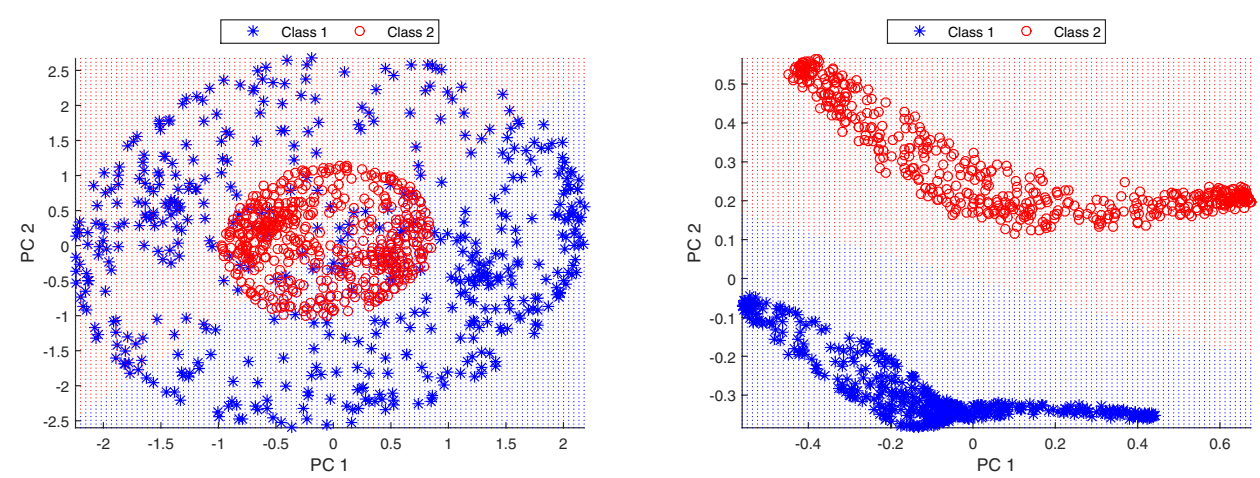

Figure 2: Visualization of Wang synthetic data set by ordinary PCA (left) and kernel PCA (right).

depicts a blue and red balls for first and second classes, respectively. The figure shows that the data is linearly inseparable. If the data is classified using Fisher linear discriminant analysis, the level of misclassification is 44.90 percent.

Data set was then reduced using ordinary PCA to obtain the first two principal components, namely PC1 and PC2 to be visualized. Visualization result is shown in Figure 2 (left), which is not much different from the original data. Data visualization with PCA remains linearly inseparable. If PC1 and PC2 are classified using Fisher linear discriminant analysis, it provides the level of misclassification by 46.50 percent. However, visualization by using kernel PCA of $\sigma=0.9153$ entirely separated the data as depicted by Figure 2 (right).

\subsection{Visualization of SCCTS Data Set}

As Wang synthesis data set, we classified the data using Fisher linear discriminant and provide the level of misclassification by 0.83 percent. It means that the data is linearly 

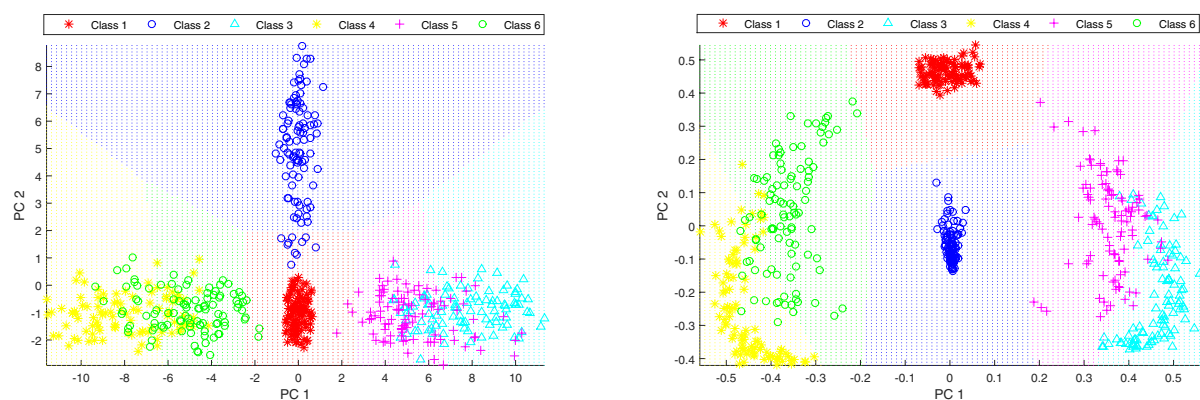

Figure 3: Visualization of SCCTS data set by ordinary PCA (left) and kernel PCA (right).
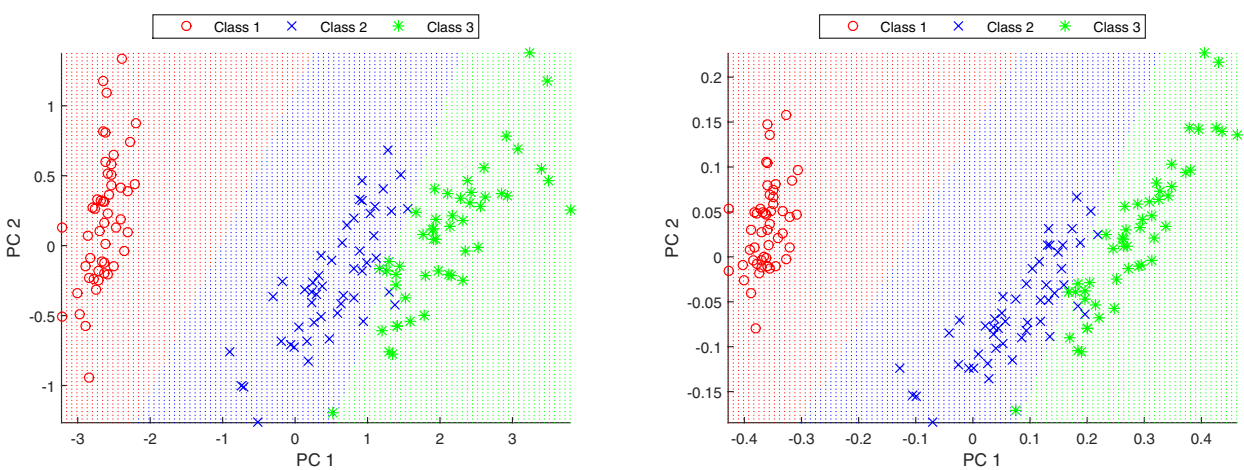

Figure 4: Visualization of Iris data set by ordinary PCA (left) and kernel PCA (right).

Table 3: Misclassification of Iris and Wine data sets

\begin{tabular}{ccccc}
\hline Data Set & Original Data & Ordinary PCA & Kernel PCA & $\sigma$ \\
\hline Iris & $2.00 \%$ & $4.00 \%$ & $2.67 \%$ & 6.8726 \\
Wine & $0.00 \%$ & $2.44 \%$ & $1.41 \%$ & 4.5673 \\
\hline
\end{tabular}

separable. However, the data cannot be visualized directly since it has 60-dimensions. Reduction by ordinary and kernel PCAs, where $\sigma=4.5301$ is applied to the later, produce the level of misclassification by 21 percent and 7 percent, respectively. The hyperplanes (1.13) are given by Figure 3.

\subsection{Visualization of Real World Data Sets}

In this part, we applied the proposed method of selecting $\sigma$ for two data sets from real world: Iris data set and Wine data set. First, the data was reduced by ordinary PCA and then by kernel PCA to compare their apparent error rates. The visualization results are shown in Figures 4 and 5, and their corresponding level of misclassification are given in Table 3. 

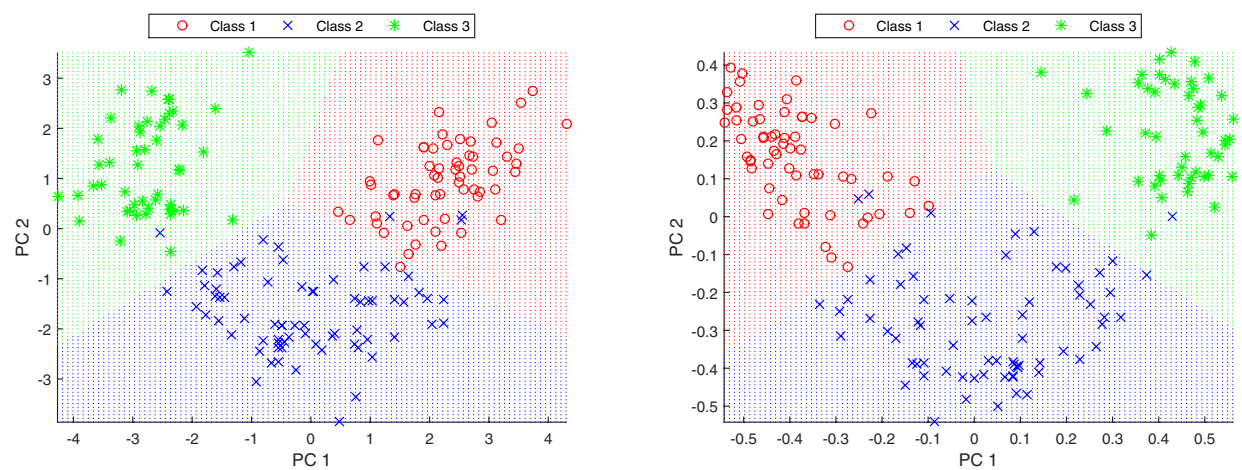

Figure 5: Visualization of Wine data set by ordinary PCA (left) and kernel PCA (right).

\section{Conclusion}

In this paper, we have discussed the theory of kernel PCA and Fisher linear discriminant analysis and made empirical studies to visualize data set using ordinary and kernel PCAs with two synthesis data sets and two real-world data sets. We have assessed the visualization of the data by ordinary and kernel PCAs under proposed method by considering the level of misclassification. We found that the value of $\sigma$ that minimizes the misclassification can be selected in the interval [min $\left.\left\|\mathbf{x}_{i}-\mathbf{x}_{j}\right\|, \max \left\|\mathbf{x}_{i}-\mathbf{x}_{j}\right\|\right]$ and with the selection of appropriate parameter $\sigma$, kernel PCA may work better than ordinary PCA because it can provide visualization of the classified data with relatively smaller misclassification.

\section{References}

[1] M.A. Alam and K. Fukumizu, "Hyperparameter selection in kernel principal component analysis," Journal of Computer Science, 10(7):1139-1150, 2014, doi:10.3844/jcssp.2014.1139.1150.

[2] R. Alcock, "Synthetic control chart time series data set," UCI Machine Learning Repository, http://archive.ics.uci.edu/ml/machine-learning- databases/ synthetic_control-mld/synthetic_control.data, 1999.

[3] C.M. Bishop, Pattern Recognition and Machine Learning, Cambridge: Springer, 2006.

[4] C. Chatfield, Problem Solving: A Statistician's Guide, 2nd Ed., Washington DC: Chapman and Hall, 1995.

[5] R.A. Fisher, "Iris plants database," UCI Machine Learning Repository http:// archive.ics.uci.edu/ml/machine-learning-databases/iris/iris.data, 1998. 
[6] M. Forina and S. Aeberhard, "Wine recognition data," UCI Machine Learning Repository, http://archive.ics.uci.edu/ml/machine-learning-databases/ wine/wine.data, 1991.

[7] R.A. Johnson RA and D.W. Wichern, Applied Multivariate Statistical Analysis, 6th Ed., Upper Saddle River, New Jersey (07458): Pearson-Prentice Hall, 2007.

[8] I.T. Jolliffe, Principal Component Analysis, 2nd Ed., New York: Springer-Verlag, 2002.

[9] K.W. Jörgensen and L.K. Hansen, "Model selection for gaussian kernel PCA denoising," IEEE Transactions on Neural Networks and Learning Systems, 23(1):163-168, 2012, doi:10.1109/TNNLS.2011.2178325.

[10] A.A. Nielsen and M.J. Canty, "Kernel principal component analysis for change detection," Image and Signal for Remote Sensing, 16(7109), 2008, doi:10.1117/12.800141.

[11] Y. Rathi, S. Dambreville and A. Tannenbaum, "Statistical shape analysis using kernel PCA," Image Processing: Algorithms and Systems, Neural Networks, and Machine Learning, SPIE, 60641B, 2006.

[12] B. Schölkopf, A.J. Smola and K.R. Muller, "Nonlinear component analysis as a kernel eigenvalue problem," Neural Computation, 10(5):1299-1399, 1998.

[13] B. Schölkopf and A.J. Smola, Learning with Kernels. Cambridge: The MIT Press, 2002.

[14] Q. Wang, "Kernel principal component analysis and its applications in face recognition and active shape models," Cornell University Library, arXiv:1207.3538v3, 2014.

[15] Q. Wang, "Synthetic data, kernel PCA and preimage reconstruction," Matlab Central, http://www.mathworks.com/matlabcentral/fileexchange/downloads/ 58633/akamai/ kPCA_v3.1.zip/kPCA_v3.1/demo1/SyntheticData.mat, 2014.

[16] D. Widjaja, C. Varon, A.C. Dorado, J.A.K. Suykens and S.V. Huffel, "Application of kernel principal component analysis for single lead ECG-derived respiration," IEEE Transactions on Biomedical Engineering, 59(4):1169-76, 2012, doi: 10.1109/TBME.2012.2186448. 